\title{
How can a Crack Opening be Extracted from a Continuous Damage Finite Element Computation? Application forthe Estimation of Permeability
}

\author{
M. Choinska*-F. Dufour**_ G. Pijaudier-Cabot***- \\ A. Huerta****_A. Khelidj* \\ * GeM, UMR 6183, IUT de Saint-Nazaire, France. \\ \{Marta.Choinska, Abdelhafid.Khelidj\}@univ-nantes.fr \\ ** GeM, UMR 6183, Ecole Centrale de Nantes, France. \\ Frederic.Dufour@ec-nantes.fr
}

*** LFC, UMR 5150, Université de Pau et des Pays de l'Adour, France.

Gilles.Pijaudier-Cabot@univ-pau.fr

**** Lab. de Càlcul Numèric, Univ. Politècnica de Catalunya, Barcelona, Spain. Antonio.Huerta@upc.es

\begin{abstract}
The assessment of the diffuse damage and the crack opening are key parameters for estimating the integrity and the strength of concrete structures in the face of environmental attacks ruled by diffusion and/or Darcy's transfers. Continuum enhanced approaches to concrete fracture are able to represent diffuse damage, crack initiation and propagation. They regard damage process as an ultimate consequence of a gradual loss of material integrity. These models, however, do not predict the crack opening. Therefore, we propose to compute the crack opening using cinematic equivalence at failure between a continuous and a discontinuous description. For this purpose, we consider a discontinuous displacement field with a given jump from which we compute the equivalent non-local strain. From a classical finite element computation we obtain a distribution of the non-local strain provided by an integral or gradient damage model. The displacement jump is computed assuming that both non-local strains are equal at the location of a crack. Finally, using this displacement jump, permeability of a cracked concrete may be determined with Poiseuille's law. This approach will be validated against experimental results on loaded discs in Brazilian splitting test.
\end{abstract}

KEY WORDS: Crack opening, damage model, strong discontinuity, fracture, permeability. 


\section{Introduction}

Transport properties of concrete, like permeability or diffusivity, are particularly important in the case of structures with a role as a hermetic, for instance containment vessels in French nuclear power plants. Evaluation of their gas tightness is critical during their service life, where concrete remains at most microcracked, but also during minor accidents, when macrocracks may appear locally. Results of experimental tests (Choinska et al., 2007), performed on concrete cylinders subjected to compressive loading, emphasize gas permeability increase upon loading. Furthermore, several regimes of permeability evolution, related to the mechanical response of concrete yielding in different cracking regimes (microcracking or macrocracking), are observed. Relationships between permeability and diffuse microcracking, described by damage variable, have already been derived theoretically and investigated experimentally (Picandet et al., 2001). The phenomenological models are most often exponential or power type with validity up to moderate diffuse damage. However, they are not valid for a cracked concrete where permeability depends essentially on macrocrack(s) opening and is governed by Poiseuille's law. In order to be complete and useful in serviceability analyses, failure analysis should therefore not only be capable of describing the distributed damage, but also it should provide crack opening.

Continuum enhanced damage models are capable of representing diffuse damage, crack initiation and propagation. They regard damage process as an ultimate consequence of a gradual loss of material integrity. These models, however, do not predict the crack opening. Another approach to model failure consists of representing the discontinuity within the material explicitly (e.g. cohesive crack model). These models relate the crack opening to the stress level and are based on the linear elastic (or plastic) fracture mechanics. They are usually used when the fracture process zone (FPZ) size is negligible compared to the structure size. Instead of trying to bridge these two classes of models as done by Mazars and PijaudierCabot (1996), we use the non-local damage model to compute the initiation and propagation of the diffuse damage and extract the discrete key parameter, i.e. the crack opening, using the concept of strong discontinuity introduced by Simo et al. (1993).

In this paper we first recall the non-local damage models (integral and gradient), then the strong discontinuity approach, and finally we apply our procedure of crack opening extraction to a tension bar where the crack location is fixed at the centre. 3D extension is not presented in this contribution, but as long as the location of the crack is known, our procedure may be applied orthogonally to the crack faces. We hope that using the crack opening extracted from a damage modelling, permeability of a cracked concrete may be determined with Poiseuille's law. In order to validate our theoretical approach, an experimental study is carried out. The device developed makes it possible to obtain complete displacement field as well as permeability evolution upon tension in the Brazilian splitting test. Comparison between 
experimental and numerical results of the splitting test will make it possible to estimate the quality of the proposed modelling.

\section{Non-local damage approach}

\subsection{Damage model}

The scalar isotropic damage model provided by Mazars (1984) is used in our study. In this model the stress $(\sigma)$ - strain $(\varepsilon)$ relationship is expressed: $\sigma=(1-D) E \varepsilon$, where $D$ is the damage scalar variable. In the case of a uniaxial tensile loading, the damage model used hereafter is based on the following damage evolution law:

$D=1-\frac{Y_{D 0}\left(1-A_{t}\right)}{Y}-\frac{A_{t}}{e^{\left[B_{t}\left(Y-Y_{D 0}\right)\right]}}$ where $\mathrm{A}_{\mathrm{t}}, \mathrm{B}_{\mathrm{t}}$ and $\mathrm{Y}_{\mathrm{D} 0}$ are model parameters and $Y$ is defined by: $Y=\max \left(Y, \varepsilon_{e q}\right)$ with $\mathrm{Y}=\mathrm{Y}_{\mathrm{D} 0}$ initially, and the equivalent strain defined as:

$\varepsilon_{e q}=\sqrt{\sum_{i=1}^{3}\left\langle\varepsilon_{i}\right\rangle_{+}^{2}}$

$\left\langle\varepsilon_{i}\right\rangle_{+}$denotes the positive part of the principal strains.

In this formulation damage depends only on the strain state at a point under consideration. Hence, this local formulation exhibits spurious strain localization. Consequently, numerical simulations yield a pathological mesh dependency and physically unrealistic results are obtained. Nevertheless, regularization techniques based on integral or gradient formulation make it possible to spread localized strain.

\subsection{Integral formulation}

In this approach, the effective strain (Eq. (1)) is substituted by the equivalent strain $\bar{\varepsilon}_{e q}$ in the equations governing the growth of damage (Pijaudier-Cabot and Bazant, 1987):

$$
\bar{\varepsilon}_{e q}=\frac{\int_{\Omega} \phi(x-s) \varepsilon_{e q}(s) d s}{\int_{\Omega} \phi(x-s) d s}
$$


Several weight functions exist in the literature, of which we choose at most often used, i.e. the Gaussian function $\phi_{G}(x-s)=e^{-\left(\frac{2\|x-s\|}{l_{c}}\right)^{2}}$ used, i.e. the Gaussian function $\phi_{G}(x-s)=e^{\left(l_{c}\right)}$ where $l_{c}[\mathrm{~m}]$, the internal length of the material, is related to the material heterogeneity parameter quantifying the non-local interactions.

\subsection{Gradient formulation}

The second class of regularized model used in this paper follows the same principle. The non-local equivalent strain $\bar{\varepsilon}_{\text {eq }}$ replaces its local counterpart. It is defined as $\bar{\varepsilon}_{e q}=\sqrt{\sum_{i=1}^{3}\left(\left\langle\bar{\varepsilon}_{i}\right\rangle_{+}\right)^{2}}$, where $\bar{\varepsilon}$ is a regularized strain distribution that is the solution of a Fredholm equation: $\bar{\varepsilon}-c \nabla^{2} \bar{\varepsilon}=\varepsilon$ in which $\sqrt{c}$ [m] can be regarded as the characteristic length of the model, related to the material heterogeneity parameter.

\section{Non-local strong discontinuity}

Let us consider the case of a 1D bar loaded in tension. The bar of length $L$ oriented by $\vec{x}$ is clamped at $\mathrm{x}=0$ and a constant velocity $v$ is applied at the other end $(\mathrm{x}=\mathrm{L})$. For simplicity, the crack location is assumed to be known in our approach (at $\mathrm{x}=\mathrm{x}_{0}$, see Figure 1(a)). In the course of loading, the bar splits into two pieces which are now assumed to be free of load.
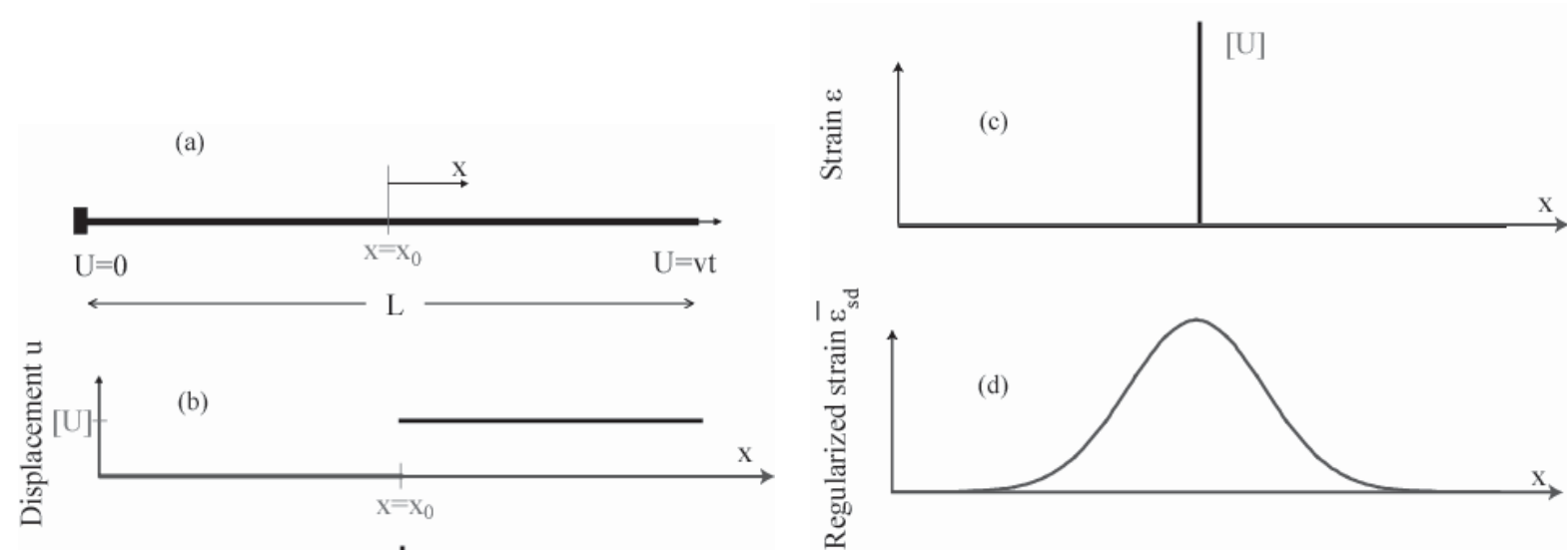

Figure 1: Strong discontinuity approach. (a) Loading system of a 1D bar with a default at $x=x_{0}$ and corresponding displacement field (b), strain field (c) and regularized strain (d)

Therefore, after failure the displacement profile (see Figure 1(b)) is described according to a strong discontinuity formulation: 
$u_{s d}\left(x, x_{0}\right)=[U] H_{\Gamma}\left(x-x_{0}\right)$

where subscript ${ }_{s d}$ stands for strong discontinuity, $\mathrm{H}_{\Gamma}$ is the Heaviside function and $[U]$ is the displacement jump across the crack. From Eq. (3), we may derive the strain field (see Figure 1(c)) with the classical symmetric gradient operator $\nabla^{s}$ :

$$
\varepsilon_{s d}=\nabla^{s} u_{s d}=[U] \delta\left(x-x_{0}\right)
$$

where $\delta(x)$ is the Dirac function. A non-local strain is then associated with the strain field (see Figure 1(d)) according to the same procedure (see Eq. (2)) used in the nonlocal damage model:

$$
\bar{\varepsilon}_{s d}\left([U], x, x_{0}\right)=\frac{\int_{\Omega} \phi(x-s) \varepsilon_{s d}\left(s, x_{0}\right) d s}{\int_{\Omega} \phi(x-s) d s}
$$

where $\phi(x)$ is the weight function. Substituting Eq. (4) into Eq. (5), and using the properties of the Dirac function, we get:

$$
\bar{\varepsilon}_{s d}\left([U], x, x_{0}\right)=\frac{[U] \phi\left(x-x_{0}\right)}{\int_{\Omega} \phi(x-s) d s}
$$

The key idea is now to compare the regularized strain fields due to the continuum formulation $\bar{\varepsilon}_{e q}\left(x, x_{0}\right)$ and the discontinuous (strong discontinuity) formulation $\bar{\varepsilon}_{s d}\left(x, x_{0},[U]\right)$ (Dufour et al., 2007). The only unknown $[U]$, the amplitude of the discontinuity - the crack opening, is computed by ensuring both regularized strains are equal at the crack location $\left(\right.$ at $\left.\mathrm{x}=\mathrm{x}_{0}\right)$ :

$$
[U]=\frac{\bar{\varepsilon}_{e q}\left(x_{0}, x_{0}\right) \int_{\Omega} \phi\left(x_{0}-s\right) d s}{\phi(0)}
$$

Both entire strain fields can be compared by defining a relative error $\Delta^{\mathrm{r}}$ :

$$
\Delta^{r}\left(x_{0},[U]\right)=\frac{\int_{\Omega}\left\|\Delta\left(s, x_{0},[U]\right)\right\| d s}{\int_{\Omega} \bar{\varepsilon}_{e q}\left(s, x_{0}\right) d s}
$$


We now apply this scheme to computations performed with the both regularized damage models (integral and gradient) for which we choose the same procedure by setting $\phi=\phi_{G}$.

\section{Numerical comparison}

We numerically solve the problem defined in Figure 1 (a) for a unit length bar and we force the crack position by setting a weak element at the centre with a smaller Young modulus. The material parameters of the damage model are: $\mathrm{E}=37,7 \mathrm{GPa}, \mathrm{E}_{\mathrm{we}}=31 \mathrm{GPa}, v=0, \mathrm{Y}_{\mathrm{D} 0}=10^{-4}, \mathrm{~A}_{\mathrm{t}}=1, \mathrm{~B}_{\mathrm{t}}=20000$.

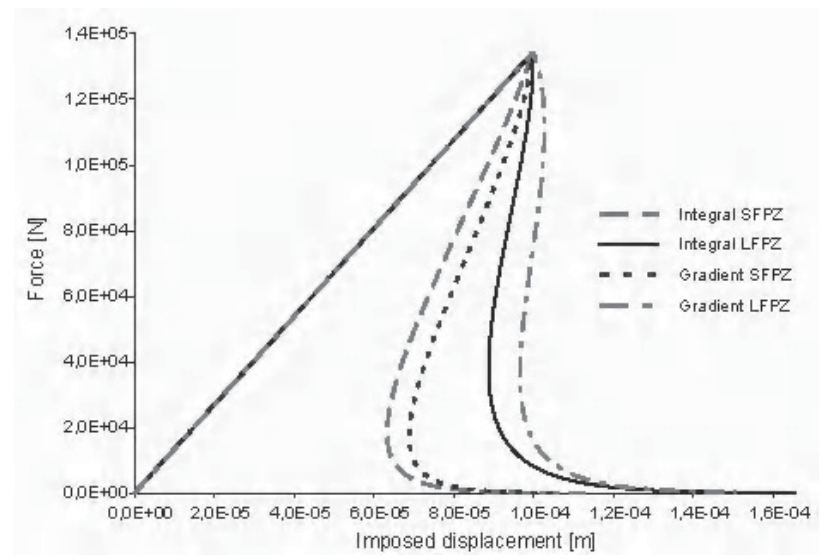

Figure 2: Global response for the two regularization techniques and two FPZ sizes
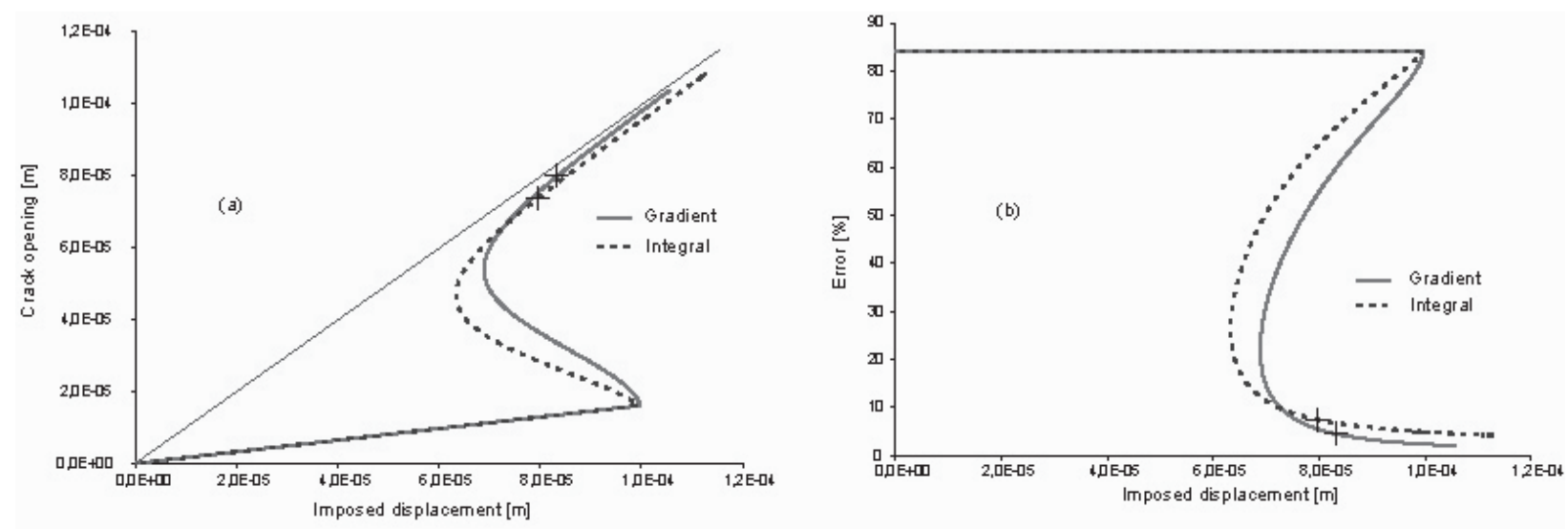

Figure 3: Crack opening as a function of imposed displacement (a); relative error as a function of imposed displacement (b). Results plotted for the smallest FPZ width

In this study two values of the internal length $1_{c}$ of the integral model are used: $0.18 \mathrm{~m}$ which gives the small FPZ (SFPZ) and $0.28 \mathrm{~m}$ which gives the large FPZ (LFPZ). The characteristic length of the gradient model $\sqrt{c}$ is calibrated to get the 
same FPZ width as for the integral model: $0.07 \mathrm{~m}$ for the small FPZ and $0.11 \mathrm{~m}$ for the large FPZ. The global responses are shown in Figure 2. As the results are qualitatively the same for any FPZ width, only the results for the smallest one are presented in Figure 3. We may observe that once the crack is fully opened, the increase of its opening is equal to the displacement applied at the active end of the bar as no further strain is stored in the material (see Figure 3(a)). Therefore the plot of the displacement jump versus the imposed displacement tends towards the bisecting line. The error (see Figure 3(b)) is quite large just after the peak load since the macro-crack is not yet clearly formed. Afterwards, the error decreases rapidly to reach a limit value of $4 \%$ for the integral approach and $2 \%$ for the gradient approach. These values represent the level of such regularized damage models ability to simulate discontinuous failure in terms of cinematic variables.

\section{Validation}

Experimental tests are performed on mortar discs loaded in compression in the diameter plane (Brazilian splitting test). This test presents the advantage of forming a single crack, the opening of which is measured precisely at the centre of the one side of a specimen using a CMOD sensor as a feedback signal during the test (see Figure 4). On the other side of the specimen measurement during loading of the displacement field by image correlation technique makes it possible to get crack opening map which will be compared to that obtained by numerical analysis based on a continuous non-local damage modelling.

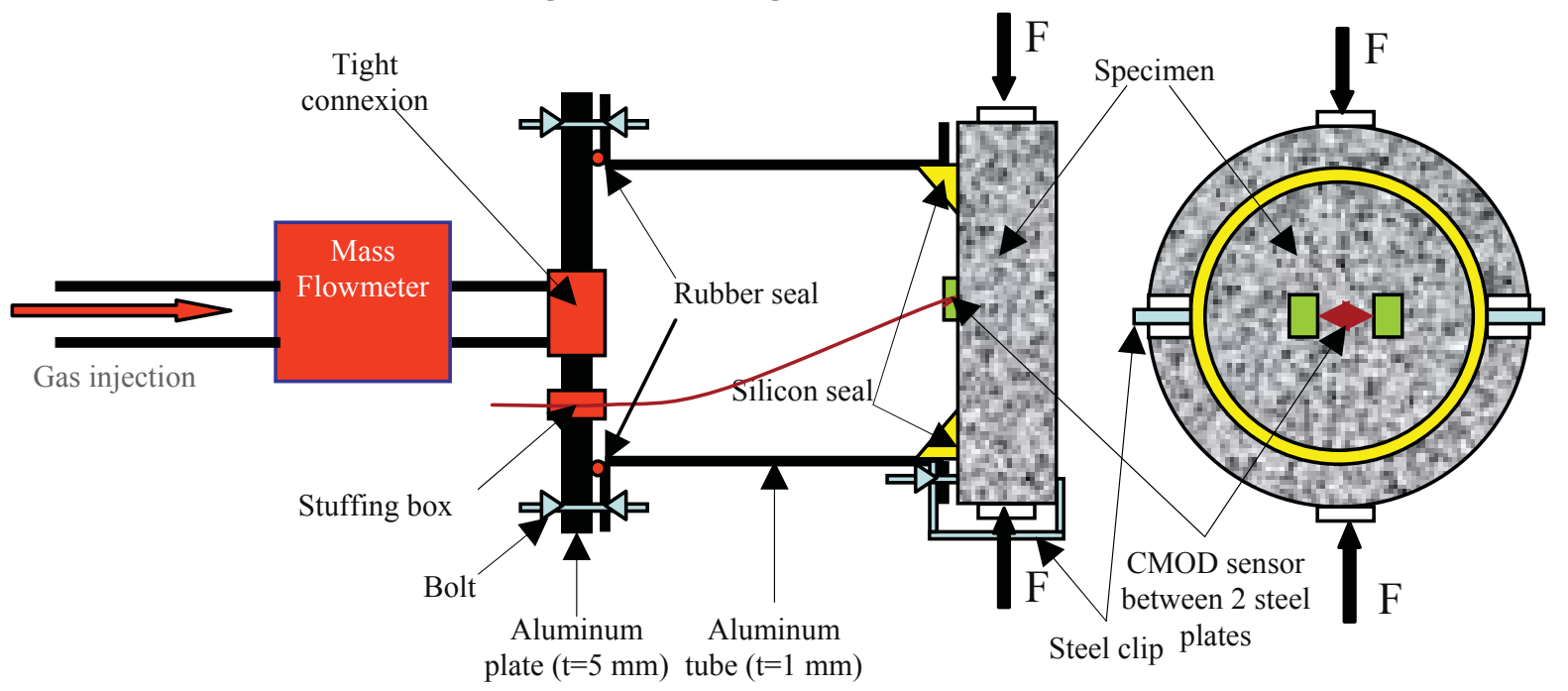

Figure 4: Diagram of the experimental device to measure crack opening and permeability during loading in the Brazilian splitting test

We explore the usefulness of the obtained results by performing permeability tests on the same discs during loading gas (see Figure 4). Permeability of a cracked disc will be finally compared with Poiseuille's permeability depending on computed crack openings. 


\section{Conclusions}

We have proposed a technique for the evaluation of a crack opening from failure analyses based on regularized damage models. Once the macro-crack if formed, our approach is able to extract a crack opening from a finite element computation with a good accuracy when the crack location is known beforehand. In order to be able to compute the crack opening when the crack location is not known, the method developed by Legrain et al. (2007) will be applied. Comparison between experimental and numerical results of the splitting test in the terms of crack opening and permeability remains the next step of this study.

\section{References}

Choinska M., Khelidj A., Chatzigeorgiou G., Pijaudier-Cabot G., "Effects and interaction of temperature and stress level related damage on permeability of concrete", vol.37, pp.79 88, Cement and Concrete Research, 2007.

Picandet V., Khelidj A. \& Bastian G., "Effect of axial compressive damage on gas permeability of ordinary and high-performance concrete", vol.31, pp.1525-1532, Cement and Concrete Research, 2001.

Mazars, J. and Pijaudier-Cabot, G., "From damage to fracture mechanics and conversely: a combined approach", vol.33, pp.3327.3342, Int. J. of Solids and Struct., 1996.

Simo, J. C., Oliver, J. and Armero, F., “An analysis of strong discontinuities induced by strain softening in rate-independent inelastic solids", vol.12, pp. 277-296, Comp. Mech., 1993.

Mazars, J., "Application de la mécanique de l'endommagement au comportement non linéaire et à la rupture de béton de structure", $\mathrm{PhD}$ thesis, Université Pierre et Marie Curie, 1984.

Pijaudier-Cabot, G. and Bazant, Z., "Non-local damage theory", vol.113, pp.1512-1533, J. of Eng. Mech., 1987.

Dufour F., Pijaudier-Cabot, G., Choinska M., Huerta A., "Extraction of a crack opening from a continuous approach using regularized damage models", submitted to Computers and Concrete, 2007.

Legrain G., Dufour F., Huerta A., Pijaudier-Cabot G., "Extraction of crack opening from a non-local damage field", Proceedings of IX International Conference on Computational Plasticity, 1, 462-465, Barcelona, Spain, 2007. 